Natural Hazards and Earth System Sciences (2002) 2: 169-179

(C) European Geosciences Union 2002

Natural Hazards
and Earth
System Sciences

\title{
Observations and modelling of snow avalanche entrainment
}

\author{
B. Sovilla and P. Bartelt \\ Swiss Federal Institute for Snow and Avalanche Research, Flüelastrasse, 11, CH-7260 Davos Dorf, Switzerland
}

Received: 20 September 2001 - Accepted: 20 December 2001

\begin{abstract}
In this paper full scale avalanche dynamics measurements from the Italian Pizzac and Swiss Vallée de la Sionne test sites are used to develop a snowcover entrainment model. A detailed analysis of three avalanche events shows that snowcover entrainment at the avalanche front appears to dominate over bed erosion at the basal sliding surface. Furthermore, the distribution of mass within the avalanche body is primarily a function of basal friction. We show that the mass distribution in the avalanche changes the flow dynamics significantly. Two different dynamical models, the Swiss Voellmy-fluid model and the Norwegian NIS model, are used to back calculate the events. Various entrainment methods are investigated and compared to measurements. We demonstrate that the Norwegian NIS model is clearly better able to simulate the events once snow entrainment has been included in the simulations.
\end{abstract}

\section{Introduction}

Since the mid-1950's simple analytical models such as the Voellmy-Salm (Salm, 1966) or the PCM model (Perla et al., 1980) have been used to calculate snow avalanche runout distances, flow velocities and impact pressures. The VoellmySalm model has been used until recently to prepare most hazard maps in Switzerland (Salm et al., 1990). Computer models are now being employed which are based on the numerical solution of the depth-averaged partial differential equations governing the mass and momentum balance of the avalanche flow (Harbitz et al., 1998).

Presently, the numerical models make many of the same simplifying assumptions of the earlier models. Clearly, one of the most limiting assumptions of the Voellmy-Salm model is that it did not account for the mass of snow that is entrained by the avalanche during its downward motion. The mass of the avalanche was considered to be constant. That is, the snow mass that starts in the release area is the same mass that arrives in the deposition zone. Recent experimen-

Correspondence to: B. Sovilla (sovilla@ slf.ch) tal observations of avalanches in Italy, clearly question this assumption (Sovilla et al., 2001).

In this paper, we first overview mass balance measurements made at the Italian Pizzac ${ }^{1}$ and Swiss Vallée de la Sionne test sites. We summarise the different experimental techniques that have been used to observe snow entrainment in field studies. We discuss the importance of the entrainment location (front or body) and how the entrained mass is eventually distributed within the avalanche. Based on the experimental observations we develop entrainment procedures and introduce them into our numerical avalanche dynamics models. Several avalanche events are then back-calculated with the models and the simulated flow velocities and heights are compared to the field observations. We also predict the flow energy consumed by snow entrainment and show that this energy is small in comparison to the total energy of the avalanche.

\section{The experimental data}

The experimental data used in this work was measured at two different experimental sites: the Swiss Vallée de la Sionne test site (Ammann, 1999) and the Italian Mount Pizzac test site (Sommavilla and Sovilla, 1998).

The most important characteristic of these data is that they are representative of two different avalanche types. In the Vallée de la Sionne test site the collected information is typical of powder and dense flow avalanches which have large dimension and develop both in an open slope and along a channelled path. In the Pizzac test site the collected information is typical of dense flow avalanches which have small dimension and flow primarily in a channelled path.

The Pizzac avalanches are either dry or wet flowing avalanches with a small or no powder part. Typical release masses are smaller than 100 tons. Arrays of mechanical flow height switches coupled with impact sensors on poles were placed at six locations along the avalanche track (see Fig. 1).

\footnotetext{
${ }^{1}$ Experimental data from Pizzac test site belongs to: ARPAV, Avalanche Centre of Arabba, Italy.
} 


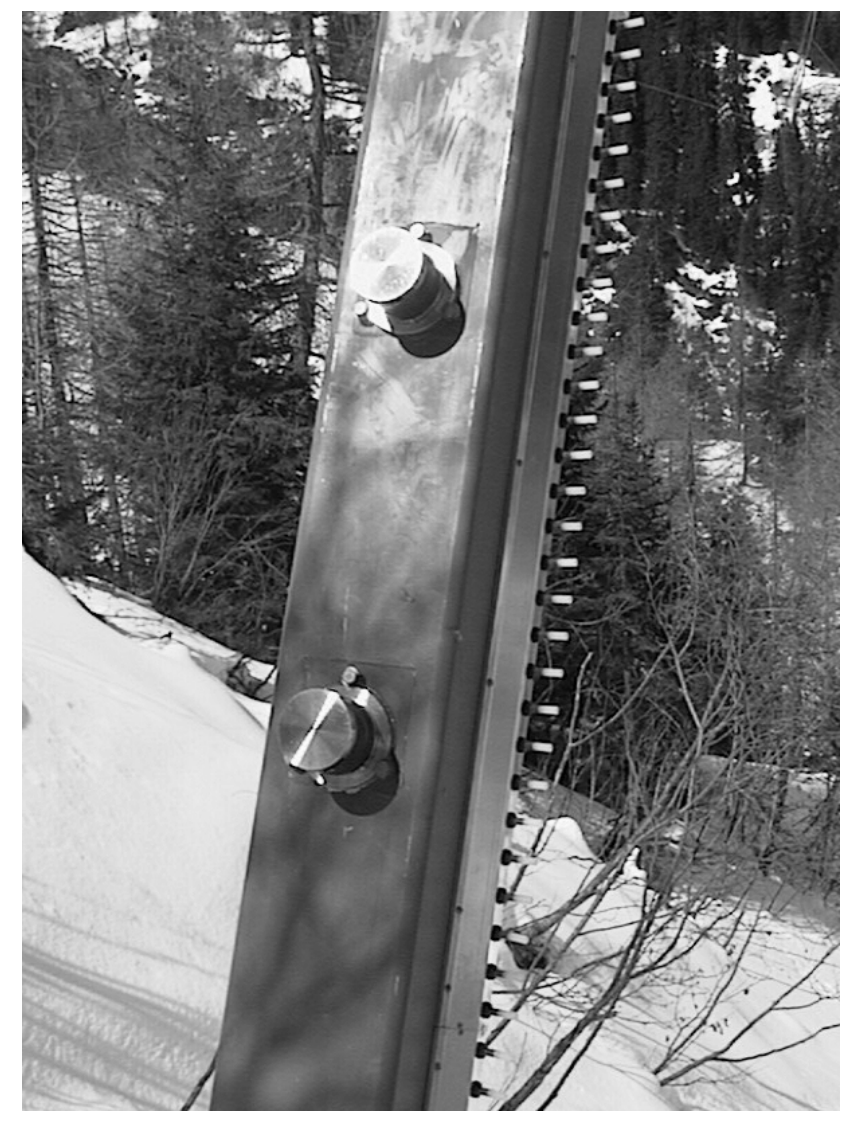

Fig. 1. Detail of flow-height measurements device showing the arrays of electro-mechanical switches spaced $5 \mathrm{~cm}$ apart. Cylindrical pressure sensors are visible too.

These instruments provide both the mean velocity along different track segments and flow depth and pressure at specific points along the avalanche path. Thus, the evolution of the avalanche can be studied. Since 1997 the avalanche mass balance has been determined for each event (Sovilla et al., 2001). Each event has been studied by analysing several sections from the starting zone to the deposition zone. At each section erosion and deposition masses have been measured. Information on evolution of the avalanche mass along the path, erosion and deposition per unit surface area $\left(\mathrm{kg} / \mathrm{m}^{2}\right)$ are available for each event.

Much larger avalanches are released at the Swiss Vallée de la Sionne (VdlS) test site. Typical release masses are of the order of several thousand tons. Since impact pressures can be as high as $500 \mathrm{kPa}$ (Schaer and Issler, 2001), any direct avalanche measurements must be made at highly reinforced obstacles. Direct measurements are therefore possible at only a few selected points along the avalanche path. The mass balance of the events is determined by photogrammetric studies (made before and after the avalanche) (Vallet et al., 2001) and video recordings. Local entrainment studies are made using three pairs of frequency modulated continuous wave radar (FMCW) (Gubler, 1984). These radars are buried into the ground and look upward. The output of these radars is

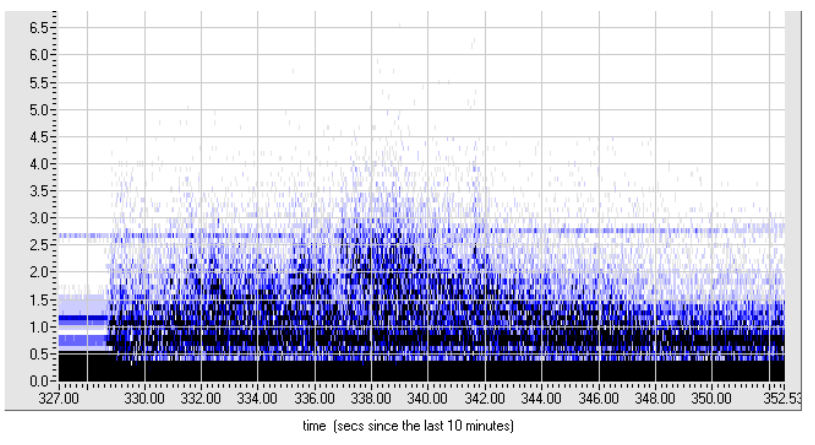

Fig. 2. FMCW radar height-intensity time plot (VDLS avalanche of 29 January 2000). The interaction between the original snow cover and the avalanche is visible (bottom left). Important information such as time, localisation and intensity of erosion can be determined. Note that maximum heights are located behind the avalanche front.

a height-intensity time plot in which both snowcover and avalanche are distinguishable (see Fig. 2). The radars provide (1) where and how much snowcover is eroded, (2) which snowpack layers have been entrained and (3) where the mass is stored in the avalanche, i.e., the avalanche shape. Manual density measurements are also made, providing it is safe to enter the avalanche test site after the avalanches. The experimental methods and recorded avalanches are summarised in Tables 1 and 2.

It should be pointed out that the variety of data and the relevant number of events has provided a better understanding of the typical behaviour of avalanches. In this paper however, we will focus on avalanche mass and entrainment process.

In the following we will use three of the avalanche events listed in Table 2. These are two Pizzac avalanches (21 December 1997 and 5 March 1999) and one large Vallée de la Sionne avalanche (25 February 1999). These events were chosen because they were the largest and best documented events (i.e. larger runout distance, mass involved in the motion, flow velocity, and pressure) recorded at the Pizzac and Vallée de la Sionne test site.

\section{Observations from the data}

\subsection{Distribution of the mass in the avalanche}

In practice models such as the Voellmy-Salm or PCM are used to prepare hazard maps. Runout distances calculated using these models are proportional to the release mass, i.e. for the same avalanche track, a larger release mass corresponds to a longer runout distance. Equal release masses should give equal runout distances. Analysis of experimental data show that real events do not always respect these rules (Sovilla et al., 2001). For example, it has been observed that avalanches having equal mass can reach different runout distances. Experimental data shows that not only the mass, but also the spatial distribution of the mass plays a fundamental role in the determination of velocities, height of flow and runout 


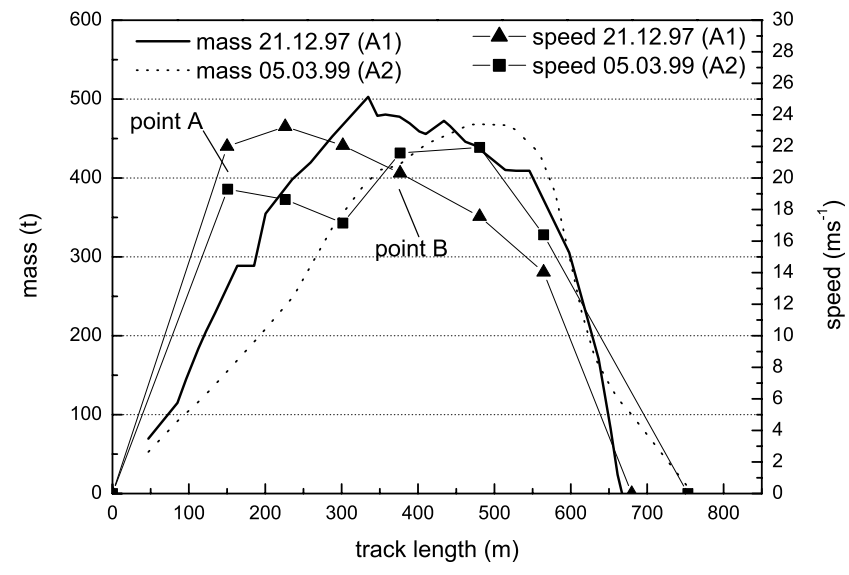

Fig. 3. Mass evolution along the avalanche trajectory and frontal speed of two Pizzac avalanches

distance. In the following we will compare two avalanche events to explain this behaviour.

Figure 3 shows the mass evolution along the avalanche trajectory as well as the frontal speed of the two Pizzac avalanches. These measurements were made at the test site Pizzac on the 21 December 1997 (avalanche A1) and on the 5 March 1999 (avalanche A2). (For more detailed information regarding the measurements, see Sommavilla and Sovilla, 1998; Sovilla et al., 2001).

It is observed that the avalanches reach nearly the same maximum mass but the avalanche $\mathrm{A} 2$ has a longer runout distance. A comparison between the two events reveals that avalanche A1 has a larger mass and a higher frontal speed along the first $400 \mathrm{~m}$ of the track. A comparison along the remaining part of the avalanche track shows different behaviours: avalanche A1 starts gradually to decelerate and deposit mass when it reaches the lower slopes; avalanche A2 continues to accelerate, increases its mass and reaches a longer runout distance.

Figure 4 shows a comparison between flow height of avalanche A1 and avalanche A2 at two positions along the avalanche path. Heights of flow are plotted as a function of time. Position A (see Fig. 3) is located at $2024 \mathrm{~m}$ a.s.l. where the slope is about $40^{\circ}$ and position B (see Fig. 3) is located at $1902 \mathrm{~m}$ a.s.l. where the slope is about $29^{\circ}$.

At position B, the frontal speeds of the avalanches and their masses are similar but avalanche A1 is decelerating while avalanche A2 is accelerating. Observation of flow shows a completely different height distribution over time. Avalanche A2 took about 13 seconds to pass through point B. Avalanche A1 took almost 26 seconds. It was characterised by lower flow heights and by an evident tail. On account of their similar masses, three hypotheses are possible: (1) the avalanche mass of A1 was distributed over a longer distance or, (2) the body of A1 was moving slower than that of A2, even thought their frontal speeds were similar or, (3) A1 was both longer and slower.

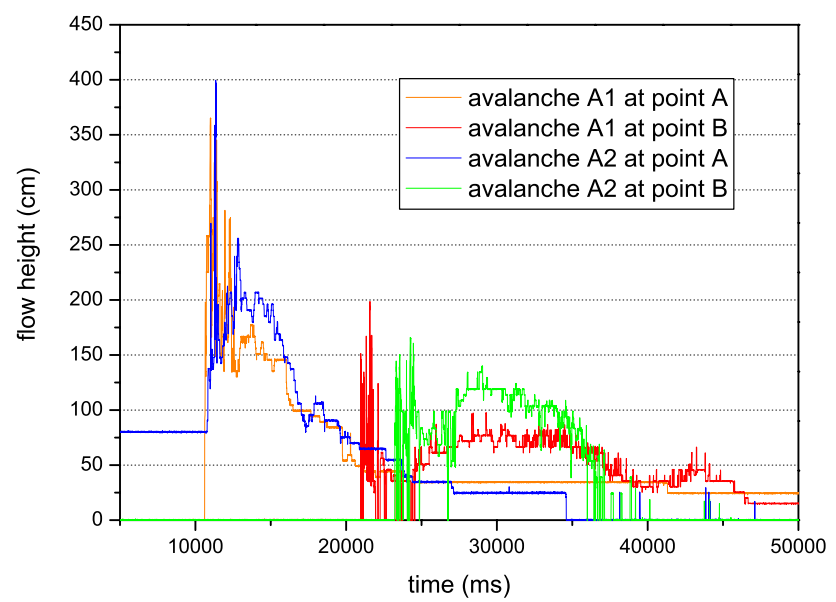

Fig. 4. Comparison between flow height of avalanche A1 and avalanche A2 at two positions along the avalanche path. Flow heights are plotted as a function of time. Position $\mathrm{A}$ is located at $2024 \mathrm{~m}$ a.s.l. where the gradient is about $40^{\circ}$ and position B is located at $1902 \mathrm{~m}$ a.s.1. where the gradient is about $29^{\circ}$.

Observations at the avalanche path immediately below point B showed that A2 deposited only half of the mass deposited by A1, suggesting that in the A1 avalanche more mass was moving slowly and, as a consequence, not contributing to the dynamics of the avalanche. This fact confirms hypothesis (3). As an intermediate result we can state that not only is the avalanche mass important, but its spatial distribution can also change the avalanche dynamics considerably. Avalanches with mass concentrated closer to the avalanche front and distributed more in height than in length tend to reach longer runout distances.

Another important observation, that corroborates this statement, concerns the distribution of the maximum heights in the avalanche. At position A, avalanche A1 has a higher frontal speed and a larger mass. Avalanche A1 reaches maximum height at the front while $\mathrm{A} 2$ reaches the maximum height about three seconds after the passage of the avalanche front. (The sharp peaks at the avalanche front result from the presence of a small powder component preceding the dense part.) At position B, where the slope angle is gentler, maximum heights are found considerably behind the front in both avalanches. It is also observed that, in each position, the faster avalanche is always characterised by a maximum value of height closer to the avalanche front.

At this point is important to understand the boundary conditions that allow the mass to move compactly, close to the avalanche front. Gubler (1986), analysing the data measured with FMCW radar, stated that high track roughness increases the snow transfer from body to tail (i.e. less avalanche height and more snow mass in the avalanche tail). The reason why avalanche A2 is moving faster and as a compact mass is exactly due to the basal sliding condition. Figure 5 shows the avalanche deposition along the avalanche trajectory, as well as an old deposit left by a previous avalanche (11 January 1999). This old deposit was characterised by a very 
Table 1. Summary of measurements and methods

\begin{tabular}{c|l|l|c|c}
\hline Measurements & Methodology & Location & VdlS & Pizzac \\
\hline \multirow{2}{*}{ Front velocity } & Image processing of video recording & All paths & $\mathrm{X}$ & \\
\cline { 2 - 5 } & From impact on poles or obstacles & At 6 locations & & $\mathrm{X}$ \\
\hline \multirow{2}{*}{ Flow Depth } & Arrays of mechanical switches & At 6 locations & $\mathrm{X}$ \\
\cline { 2 - 5 } & FMCW radars & At 3 locations & $\mathrm{X}$ & \\
\hline \multirow{2}{*}{$\begin{array}{c}\text { Mass balance } \\
\text { (Deposition } \\
\text { distribution and } \\
\text { erosion) }\end{array}$} & $\begin{array}{l}\text { Established methodology of manual } \\
\text { measurements (Sovilla et al., 2001) }\end{array}$ & $\begin{array}{l}\text { Several sections } \\
\text { along the avalanche } \\
\text { path }\end{array}$ & & $\mathrm{X}$ \\
\cline { 2 - 5 } & Photogrammetry (global mass balance) & $\begin{array}{l}\text { Before and after the } \\
\text { event, release and } \\
\text { deposition zone }\end{array}$ & $\mathrm{X}$ & \\
\cline { 2 - 5 } & FMCW radar (local mass balance) & At 3 locations & $\mathrm{X}$ & \\
\hline $\begin{array}{c}\text { Snow properties } \\
\text { (snow pit, density) }\end{array}$ & Manual measurements & $\begin{array}{l}\text { Several points along } \\
\text { the avalanche path }\end{array}$ & $\mathrm{X}$ & $\mathrm{X}$ \\
\hline
\end{tabular}

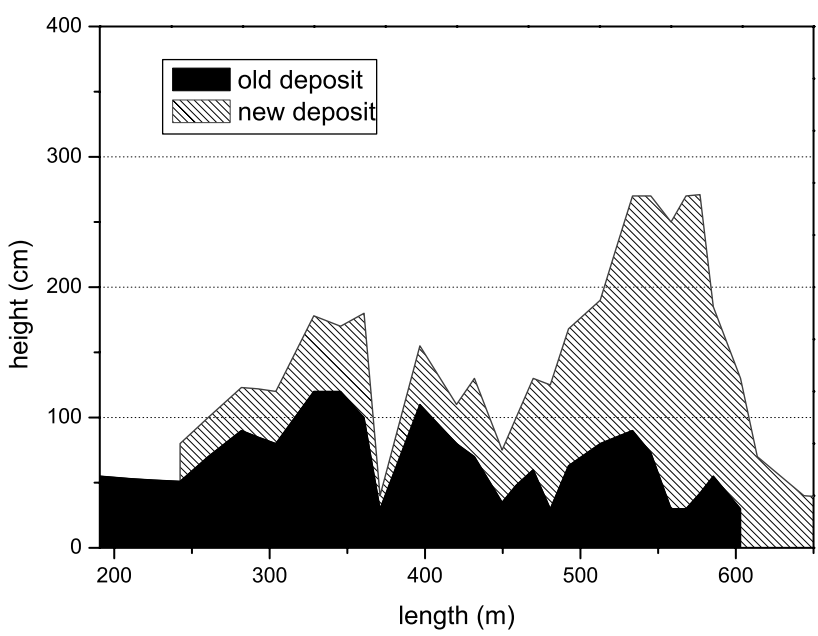

Fig. 5. Longitudinal section of the avalanche deposition along the avalanche trajectory (5 March 1999), as well as an old deposit left by a previous avalanche (11 January 1999). This older deposit was characterised by a very smooth frozen hard surface over which avalanche A2 slid.

smooth frozen hard surface over which avalanche A2 slid. The very low value of friction exerted by the slide-surface allowed the avalanche to go faster and decreased the transfer of mass to the avalanche tail. Because of its higher speed, the avalanche continued to collect mass over low slopes and reached a longer runout distance.

\subsection{Entrainment location}

The distribution of the mass within the avalanche is influenced by snow cover/ground condition. However the location of where the mass is entrained is another factor that must be taken into account. Investigation of the entrainment location is possible by analysis of the FMCW radar measurements in Vallée de la Sionne. Figure 2 shows an example of
FMCW radar output. In this figure, the amplitude of the signal reflected from different heights in the avalanche is plotted as a function of time on a three-dimensional intensity plot. A graph relating flow heights and intensities is obtained. At the bottom-left it is possible to observe how the avalanche interacts with the snow cover. This event was measured at the Vallée de la Sionne test site on the 29 January 2000. The graph shows that the avalanche entrained almost $1 \mathrm{~m}$ of snow cover immediately at the front and then followed to slide over a constant surface localised about $0.30 \mathrm{~m}$ over the ground without entraining any more mass. Similar to the Pizzac events that were studied, it is possible to observe that the maximum height and intensity of the signal are located almost 10 seconds behind the avalanche front. The fact that the erosion is located in the front, but the maximum flow height is behind the front, suggests that there is a mass transfer from the avalanche head to the avalanche body, i.e., the collected snow requires a certain amount of time before it is accelerated up to the avalanche speed.

Analysis of different FMCW measurements shows that front entrainment processes appear to dominate over basal erosion. Our observations are that avalanches tend to dive into the snow cover and slide over a more resistant and older layer or slide on the ground. A frontal impact between the avalanche front and the snow cover takes place and the avalanche collects all the snow immediately at the front. This process is often referred to as "ploughing". However, it has also been observed that, avalanches flow on a hard resistant layer within the snowcover. It has been conjectured that avalanches can scrape mass from the surface in a process termed "basal erosion".

\subsection{Conclusions from the measurements}

The following conclusions can be made from the measurements: 
Table 2. Summary of the events

\begin{tabular}{|c|c|c|c|c|c|c|c|}
\hline \multirow[b]{2}{*}{ Site } & \multirow[b]{2}{*}{ Event } & \multirow{2}{*}{$\begin{array}{l}\text { Avalanche } \\
\text { type }\end{array}$} & \multirow{2}{*}{$\begin{array}{c}\text { Release } \\
\text { Mass } \\
\text { (t) }\end{array}$} & \multirow{2}{*}{$\begin{array}{l}\text { Maximum } \\
\text { Mass } \\
\text { (t) }\end{array}$} & \multirow{2}{*}{$\begin{array}{c}\text { Average } \\
\text { speed } \\
(\mathrm{m} / \mathrm{s})\end{array}$} & \multicolumn{2}{|c|}{$\begin{array}{l}\text { Entrained snow } \\
\text { cover }\end{array}$} \\
\hline & & & & & & $\begin{array}{l}\text { Height } \\
\text { (m) }\end{array}$ & $\begin{array}{l}\text { Density } \\
\left(\mathrm{kg} / \mathrm{m}^{3}\right)\end{array}$ \\
\hline Pizzac & 05.12 .97 & Dry/Dense & 34 & 62 & 11 & 0.70 & $135-185$ \\
\hline Pizzac & 21.12 .97 & Dry/Dense & 84 & 506 & 17.9 & $1.2-0.5$ & $140-290$ \\
\hline Pizzac & 14.04 .98 & Moist/Dense & 31 & 127 & 12.3 & $0.6-0.1$ & $115-165$ \\
\hline Pizzac & 28.04 .98 & Wet/Dense & 90 & 297 & 7.6 & $0.3-0.05$ & 500 \\
\hline Pizzac & 05.03 .99 & Dry/Dense & 53 & 468 & 17.6 & 0.73 & $160-320$ \\
\hline VdlS & 30.01 .99 & Dry/Powder & $\sim 12000$ & - & $70^{*}$ & $\sim 1.2$ & $180-290$ \\
\hline VdlS & 10.02 .99 & Dry/Powder & $\sim 18700$ & - & $57^{*}$ & $\sim 1.5$ & $\sim 200$ \\
\hline VdlS & 25.02 .99 & Dry/Powder & $\sim 80000$ & - & $70^{*}$ & $\sim 1.5$ & $\sim 200$ \\
\hline
\end{tabular}

* maximum speed

(1) The distribution of the mass in the avalanche body can strongly influence the dynamic of the avalanche.

(2) The distribution of mass in the avalanche is dependent on the entrainment location as well as how the mass is transferred within the avalanche.

(3) The distribution of the mass within the avalanche is dependent on the terrain/snow cover friction. Lower friction decreases the snow transfer from the body to tail of the avalanche. This conclusion corroborates the observations of Gubler (1986).

(4) A comparison between two avalanches of equal mass shows that the avalanche with more mass concentrated at the avalanche front has higher velocity and reaches longer runout distance.

(5) Avalanches with an evident tail deposit mass along the avalanche path, suggesting that the deposition process begins at the tail, where the velocity is smaller.

(6) For flowing avalanches the maximum flow heights are generally located considerably behind the front. However, when the avalanche runs on a steep track (35$40^{\circ}$ ), the maximum heights move forward, closer to the avalanche front.

(7) Maximum impact pressures correspond to the maximum height position. Exceptions are due to the presence of a powder cloud moving at the front of the avalanche. In this case, particles exiting the flowing core can cause high punctual impact pressures.

(8) Front entrainment (ploughing) processes appear to dominate over basal erosion. However, which process dominates is a function of the snow cover characteristics and avalanche dimension.
(9) The entrained snow does not reach the avalanche velocity instantaneously. The time delay causes maximum flow heights to be located behind the avalanche front.

\section{Model equations}

Depth-averaged continuum models are used to predict avalanche runout distances, flow heights and velocities given the initial starting mass and shape, the values of physical parameters and the terrain coordinates of the avalanche path. The models numerically solve (Sartoris and Bartelt, 2000) the mass (volume) and momentum balance equations:

$$
\begin{aligned}
& \frac{\partial A}{\partial t}+\frac{\partial Q}{\partial x}=\dot{S}_{e}-\dot{S}_{d} \\
& \frac{\partial Q}{\partial t}+\frac{\partial}{\partial x}\left[\alpha \frac{Q^{2}}{A}\right]=A\left[g F_{0}-g F_{f}-F_{e}\right]-\lambda g A \frac{\partial h}{\partial x} \cos \varphi(2)
\end{aligned}
$$

where $x$ is the length along the avalanche path; $t$ is the time; $g$ the acceleration due to gravity; $A(x, t)$ is the cross-sectional flow area given by

$A(x, t)=w(x) \cdot h(x, t)$,

where $w(x)$ is the known flow width and $h(x, t)$ is the avalanche flow height. $Q(x, t)$ is the depth-averaged discharge,

$Q(x, t)=A(x, t) \cdot U(x, t)$.

$U(x, t)$ is the flow velocity. The right-hand side of the mass equation contains the terms $\dot{S}_{e}$ and $\dot{S}_{d}$ and which are the volumetric snow entrainment and deposition rates. The mass rates are found by multiplying $\dot{S}_{e}$ and $\dot{S}_{d}$ and with the avalanche density $\rho_{f}$, which is assumed to be constant. The right-hand side of the momentum equation contains the gravitational acceleration $g F_{0}(g \sin \varphi)$ along the track segment with inclination $\varphi(x) . F_{f}$ is the flow friction and $F_{e}$ is the 


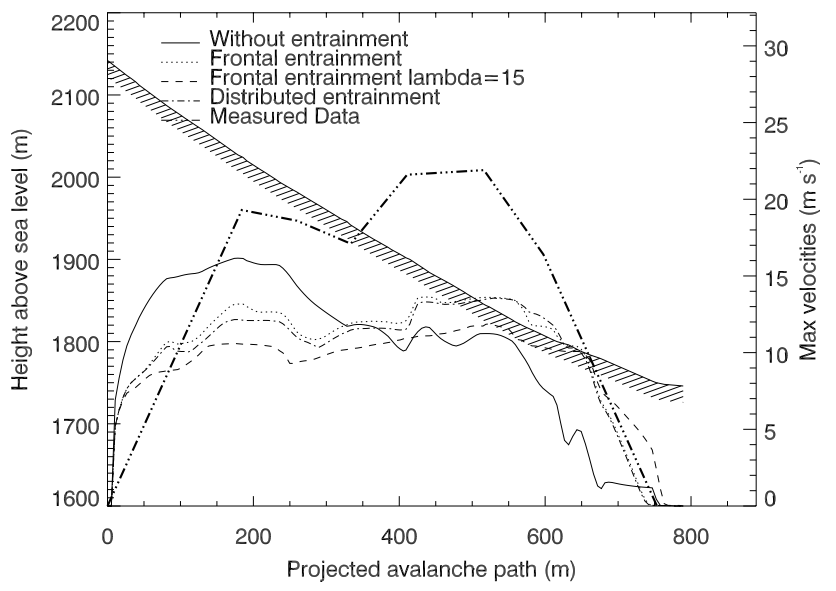

Fig. 6. Speed simulations of a small avalanche (Pizzac event of 5 March 1999) using the Voellmy-fluid model. Simulations without entrainment, with frontal entrainment $(\lambda=2.5$ and $\lambda=15)$ and with entrainment distributed along the avalanche length are compared with experimental data. Note that calculated speeds are too low.

entrainment friction. $F_{f}$ will be discussed below for both a Swiss Voellmy-fluid (Bartelt et al., 1999) and Norwegian NIS model (Norem et al., 1989; Harbitz et al., 1998). $F_{e}$ is the avalanche deceleration due to (1) fracture of the snow cover in front of the avalanche, (2) acceleration of the fracture mass up to the avalanche flow velocity and (3) raising the entrained snow mass up to the mean flow height. We will consider that the deceleration needed to fracture the snow cover is negligible in comparison to the deceleration caused by accelerating the entrained mass up to the avalanche velocity.

The energy required to accelerate the entrained mass $\Delta m_{e}$ over a time step $\Delta t$ up to the avalanche speed is given by the relation:

$E c=\frac{1}{2} \Delta m_{e} U^{2}$.

The change in mass is calculated according to:

$$
\Delta m_{e}=w(x) \cdot h_{s}(x) \cdot U(x, t) \cdot \Delta t \cdot \varphi
$$

where, $h s(x)$ and $\varphi$ are the height and density of the entrained snowcover. Calculating expression (6) for a real avalanche event (for instance: small avalanche normal values: $U(x, t)=20 \mathrm{~m} \mathrm{~s}^{-1}, w(x)=10 \mathrm{~m}, h_{s}(x)=0.4 \mathrm{~m}, \rho=$ $\left.300 \mathrm{~kg} / \mathrm{m}^{3}, \Delta t=0.005 \mathrm{~s}\right)$, we obtain:

$\Delta m_{e}=10 \cdot 0.4 \cdot 20 \cdot 0.005 \cdot 300=120 \mathrm{~kg}$

Considering that the mass of a small avalanche is of the order of 34000-90000 kg in the release zone and can reach $500000 \mathrm{~kg}$ (see Table 2), the energy required to accelerate the increment of mass to the avalanche speed is very small in comparison to the energy of the avalanche (order of $10^{3}$ larger). In the same way it can be demonstrated that the deceleration caused by raising the entrained snow mass up to

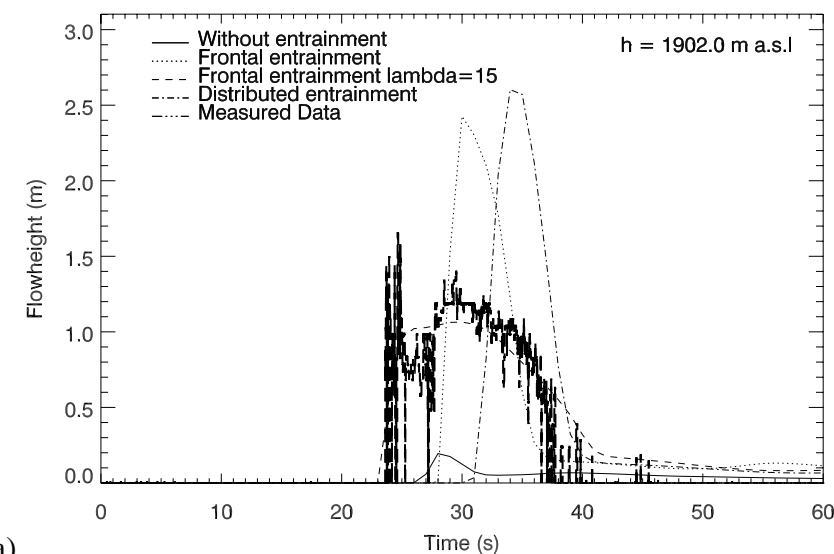

(a)

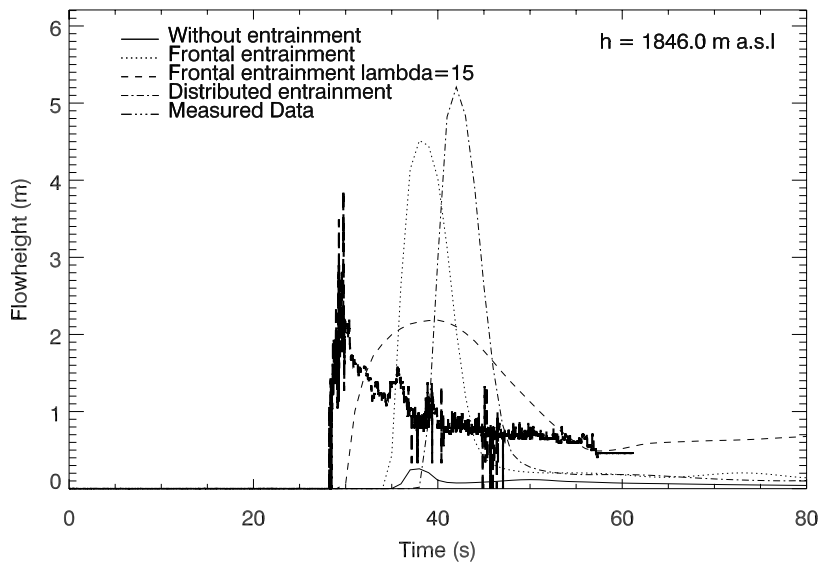

(b)

Fig. 7. Flow height simulations of a small avalanche (Pizzac event of 5 March 1999) using the Voellmy-fluid model. Simulations without entrainment, with frontal entrainment $(\lambda=2.5$ and $\lambda=15)$ and with entrainment distributed along the avalanche length are performed in two positions along the avalanche path. Calculated data are compared with experimental data. (a) shows a point located at $1902 \mathrm{~m}$ a.s.1. where the gradient is about $29^{\circ}$. (c) shows a point located at $1846 \mathrm{~m}$ a.s.l. where the gradient is about $38^{\circ}$.

the mean flow height is very small. Therefore $F_{e}$ can be neglected (see Bartelt and Stöckli, 2001) for a similar analysis with biomass entrainment loadings in forest).

The remaining two values on the left-hand side of the momentum equation are the velocity profile factor $\alpha(x, t)$ and the active-passive pressure coefficient $\lambda$. The latter parameter governs the amount of friction introduced via longitudinal straining of the flow body (see Bartelt et al., 1999).

\subsection{The Voellmy-Fluid model}

The Voellmy-Fluid model assumes no shear deformation. The flow body moves as a plug with everywhere the same mean velocity over the height of flow, i.e.

$\alpha(x, t)=1$. 
The friction slope $F_{f}$ is given by,

$F_{f}=\mu \cos \varphi+\frac{u^{2}}{\xi h}$,

where $\mu$ and $\xi$ are flow parameters depending on snow properties, track roughness and avalanche size. Although the shear deformations are zero, longitudinal straining of the flow plug exists and resistance to active (tensile) and passive (compressive) flow states is given the active/passive pressure governed by the relation:

$\left.\begin{array}{l}\lambda_{a} \\ \lambda_{p}\end{array}\right\}=\tan ^{2}\left(45^{\circ} \pm \frac{\phi}{2}\right)$

with:

$\lambda=\left\{\begin{array}{l}\lambda_{a} \text { for } \frac{\partial U}{\partial x}>0 \\ \lambda_{p} \text { for } \frac{\partial U}{\partial x} \leq 0\end{array}\right\}$

where $\phi$ is the internal friction angle. Typical values are in the range $20^{\circ} \leq \phi \leq 40^{\circ}$, leading to active/passive values in the range $0.2 \leq \lambda_{a} \leq 0.5$ and $2.0 \leq \lambda_{p} \leq 4$.6. This formulation neglects the influence of the cohesion of the snow. See Savage and Hutter (1989) for more details.

\subsection{The Norwegian Nis model}

Unlike the Voellmy-Fluid model, the shear deformation rates, $\dot{\lambda}$, in the Norwegian NIS model are nonzero,

$\dot{\lambda}=\frac{\partial U}{\partial z}=\frac{3}{2} \frac{\left(u_{h}-u_{0}\right) \sqrt{1-\frac{z}{h}}}{h}$

where $u 0$ is the velocity at the base of the avalanche and $u h$ the velocity at the top surface. Since the vertical speed profile is not constant, the velocity profile factor is given by the relation

$\alpha(x, t)=\frac{5}{4}\left[\frac{9 u_{h}^{2}+6 u_{h} u_{0}+5 u_{0}^{2}}{\left(3 u_{h}+2 u_{0}\right)^{2}}\right]$

The ratio $R$ between these velocities is:

$R=\frac{u_{h}}{u_{0}}=\left[1+\frac{2 h}{3} \sqrt{\frac{s}{\rho\left(m-b v_{2}\right)}}\right]$

$s, m, b, v_{1} v_{2}$ are the model parameters. In particular, $b$ is the coefficient of dry friction, $s$ is the velocity-squared dynamic friction coefficient, $m$ is the shear viscosity and $v_{1} v_{2}$ are the normal stress viscosities. The friction slope is given by:

$S_{f}=b \cos \varphi+\frac{s u_{0}^{2}}{\rho g h}-\frac{9 v_{1}\left(u_{h}-u_{0}\right)}{4 h^{2}} \frac{\partial\left(u_{h}-u_{0}\right)}{\partial x}$

and the passive pressure by the relation:

$\lambda=\left[1+\frac{9 v_{1}\left(u_{h}-u_{0}\right)^{2}}{8 g h^{3} \cos \varphi}\right]$

For a complete description of the model see Norem et al. (1989), Harbitz et al. (1998) or Bartelt et al. (1999).

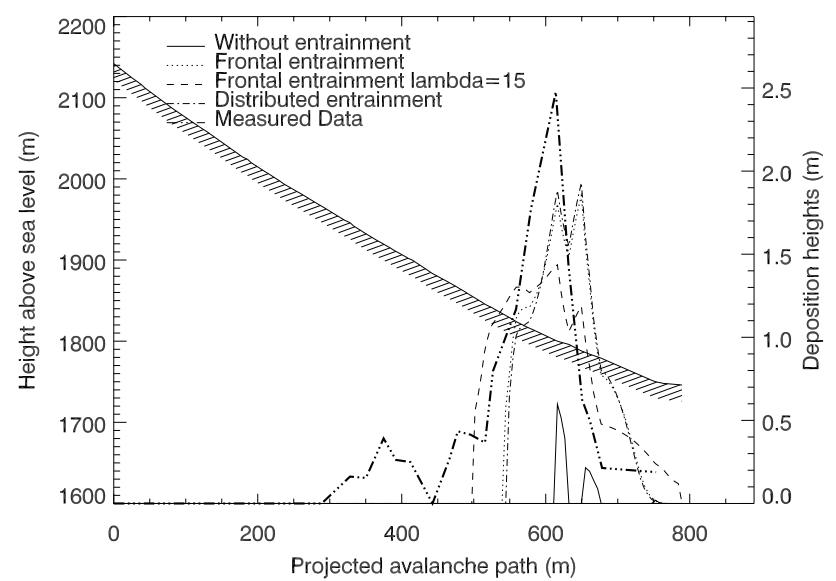

Fig. 8. Deposit simulations of a small avalanche (Pizzac event of 5 March 1999) using the Voellmy-fluid model. Simulations without entrainment, with frontal entrainment $(\lambda=2.5$ and $\lambda=15)$ and with entrainment distributed along the avalanche length are compared with experimental data. Note the difference between simulation with and without entrainment.

\subsection{Small avalanche: Pizzac Simulations}

Small avalanches with short return period, which loose mass on steep slope and quickly come to an halt (i.e. Pizzac events), are difficult to calculate with current dynamical models.

The simulation of avalanche A2 using the Voellmy-Salm model (without entrainment) shows these limits: frontal , height of flow, avalanche length and deposits are strongly underestimated $(\mu=0.35, \xi=3000$ and $\lambda=2.5)$. The speed results are shown in Fig. 6. Comparison between the measured and calculated flow heights are shown in Figs. 7a, b; deposition heights are shown in Fig. 8.

The same measurements were back-calculated using the NIS model (without entrainment). The simulation has been performed using friction values closed to the smallest values within the range specified by Norem (1989) $(m=0.005, b=$ $0.4, s=0.5, v_{1}=0.001$ and $\left.v_{2}=0.0001\right)$. Fig. 9 shows the comparison between model results and measurements. We note that calculated and measured speeds are in good agreement, as well as the runout distances. However, using constant parameters, it is not possible to obtain the acceleration measured in the second part of the track. On the contrary, flow height, avalanche length and depositions are unrealistic. (See Figs. 10a, b and 11.)

Avalanche A2 was characterised by an important erosion process. It was able to increase its mass up to 9 times with respect to the released mass. To demonstrate the mass variation effect, the model has been modified to include entrainment.

The simulated avalanche entrains snow by eroding a userspecified snow cover. The snow cover is composed of up to three layers. Each layer is characterised by a height and density equal to the height and density of the layers entrained by the real avalanche. The density values used for these calcu- 


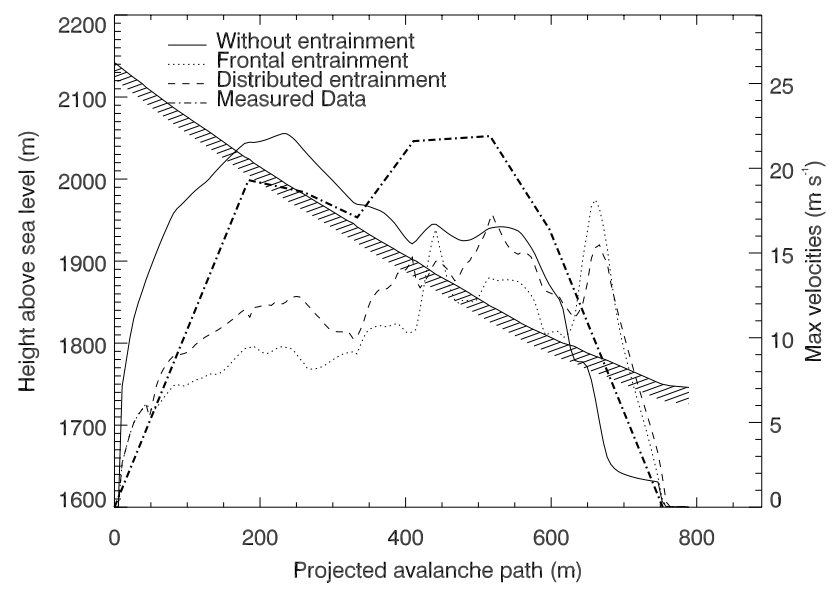

Fig. 9. Speed simulations of a small avalanche (Pizzac event of 5 March 1999) using the NIS Norwegian model. Simulations without entrainment, with frontal entrainment and with entrainment distributed along the avalanche length are compared with experimental data. Note that also if calculated speed are low, the introduction of the entrainment reproduce the avalanche acceleration observed in the second part of the track.

lations are ranging between 160 and $320 \mathrm{~kg} / \mathrm{m}^{3}$; the entrainment height between 0.4 and $0.70 \mathrm{~cm}$. All the user-specified snow cover was entrained.

Since front entrainment processes appear to dominate (see Sect. $3.3(8)$ ) in the first simulation the mass is entrained at the avalanche front. The model assumes that the eroded mass is instantaneously accelerated to the avalanche speed. A second simulation is performed in which the acceleration requires a specified amount of time (indicated in the figures as "distributed entrainment") (according to point Sect. 3.3 (9)).

The simulations results performed using the NIS model are shown in Figs. 9, 10a, b, and $11\left(m=0.055, b=0.5, s=3, v_{1}\right.$ $=1$ and $v_{2}=0.1$ ). It is observed that the parameters used in the simulation with entrainment, i.e. more mass, are higher than the parameters used in the case without entrainment. In the simulation with entrainment the calculated speed in the runout zone is higher. To stop the avalanche at the same runout distance larger friction parameters are needed.

In general, the calculations without entrainment show that the calculated speeds are lower than the experimental data and, in the first part of the track, also lower than the calculated speeds without entrainment. However, simulations with snow entrainment reproduce the avalanche acceleration observed in the second part of the track better. Recall that in this case the A2 avalanche started to accelerate in spite of the lower gradient (see Sect. 3.1 and Fig. 3).

Height of flow over steep slopes and the distribution of deposit match for both simulations the measurements (see Figs. 10a, b). Over gentle slopes, in order to reach a good agreement between measured and calculated heights, a timedelay entrainment is necessary (see Fig. 10a). Depositions and flow heights are in good agreement with measured data

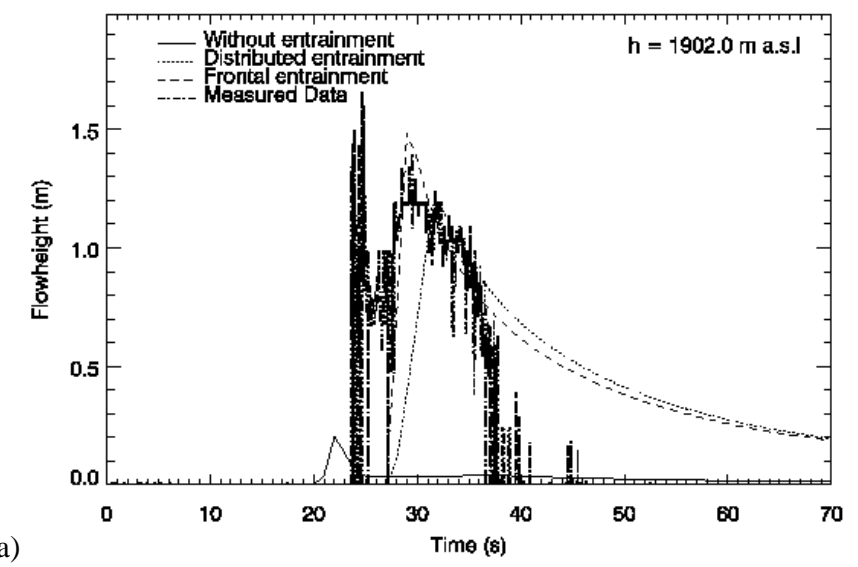

(a)

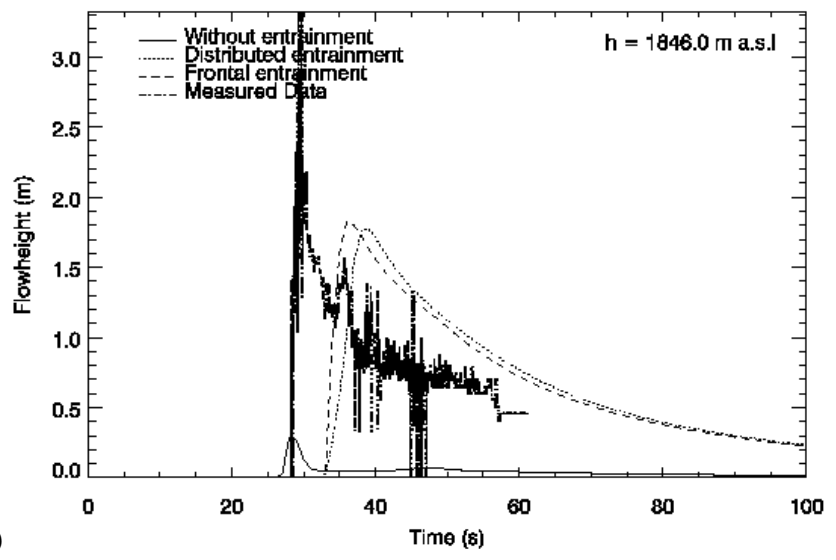

Fig. 10. Flow height simulations of a small avalanche (Pizzac event of 5 March 1999) using the NIS Norwegian model. Simulations without entrainment, with frontal entrainment and with entrainment distributed along the avalanche length are performed in two positions along the avalanche path and compared with experimental data. (a) shows a point located at $1902 \mathrm{~m}$ a.s.l. where the gradient is about $29^{\circ}$. (b) shows a point located at $1846 \mathrm{~m}$ a.s.l. where the gradient is about $38^{\circ}$. Note the very good agreement between calculated (with entrainment) and measured flow heights.

\section{too (see Fig. 11).}

It is interesting to observe that by increasing the time in which the collected snow is accelerated to the avalanche speed, maximum flow heights move back inside the avalanche body. If the time delay is too large the avalanche dynamics can change substantially (see Fig. 12). In this figure the same snow mass is entrained using three different erosion methods: frontal erosion (solid line), erosion distributed along all the avalanche length (dotted line) and erosion distributed over a part of the avalanche length (dashed line). The erosion distributed over only a part of the avalanche length (in Fig. 12 distributed entrainment) gives the best fit between experimental data and simulation.

The same simulations have been performed with the Voellmy Salm model $(\mu=0.48, \xi=1600$ and $\lambda=2.5$ ) (see Figs. 6-7a, 7b-8). The results of these simulations are not as good as the simulation performed with the NIS model. Flow heights are strongly over-estimated and, although the simu- 


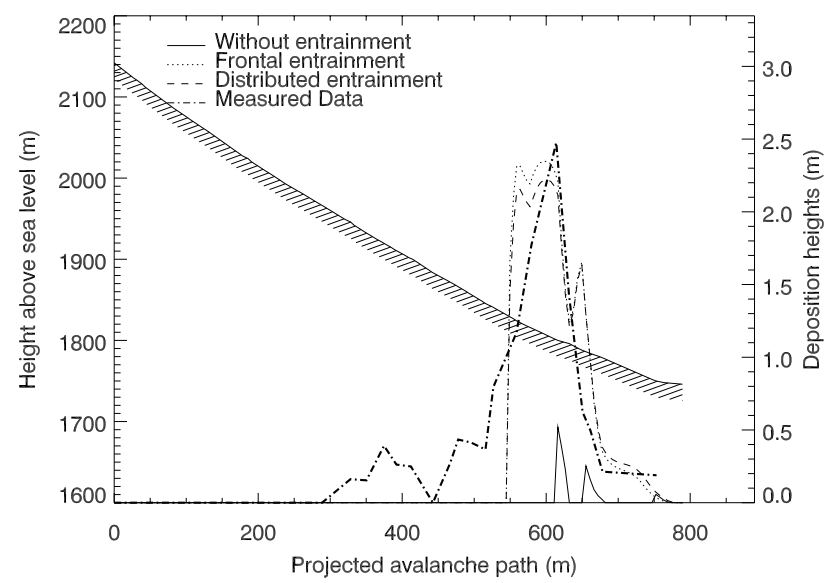

Fig. 11. Deposit simulations of a small avalanche (Pizzac event of 5 March 1999) using the NIS Norwegian model. Simulations without entrainment, with frontal entrainment and with entrainment distributed along the avalanche length are compared with experimental data.

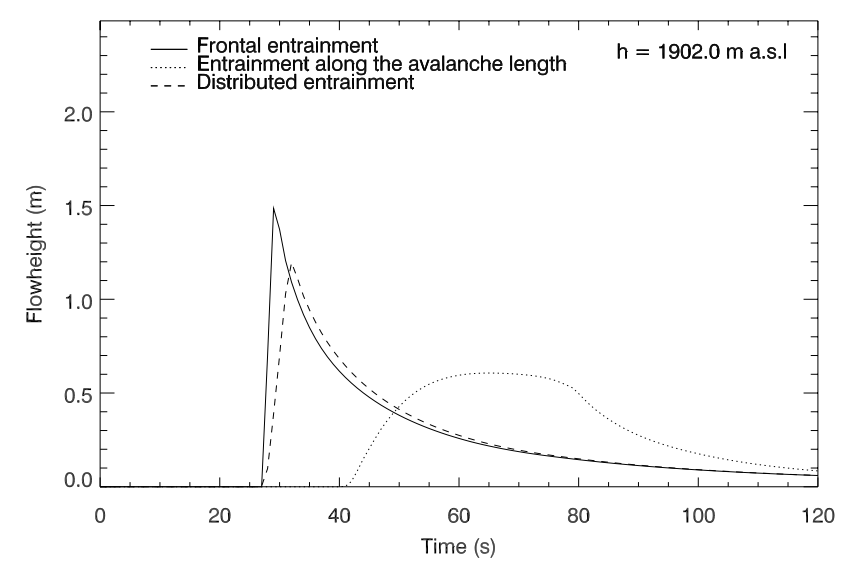

Fig. 12. Flow height simulations of the Pizzac avalanche using the NIS model. The same snow mass is entrained using three different erosion methods: frontal erosion (solid line), erosion distributed along all the avalanche length (dotted line) and erosion distributed over a part of the avalanche length (dashed line - distributed entrainment). Dashed line gives the best fit between experimental data and simulation.

lated maximum heights are behind the front (see Sect. 3.3 (6)), the mass is distributed more in the height that in length (see Figs. 7a, b). This demonstrates that the assumption of no shear deformation together with the longitudinal straining do not describe the physical phenomenon appropriately because the mass transfer to the avalanche body is not correctly modelled. To calculate correct height of flow, the $\lambda$ parameter must be increased to a value of 15 . This could be physically explained by assuming an internal friction angle of about $60^{\circ}$ or by considering the influence of the snow cohesion. However, the introduction of a high $\lambda$ value decreases the speed and increases the runout distance making the simulations incorrect.

The NIS model, which accounts for a non zero shear defor- mation rate and a longitudinal straining governed by a passive pressure depending on the shear deformation rate, describes and simulates small avalanche behaviour better.

\subsection{Large avalanche: VdlS simulation}

It has been already demonstrated that the lack of snow entrainment in the models is one of the reasons why they function poorly for small avalanches events where mass evolution is significant (Sect. 4.3). On the other hand, it has been observed that large avalanches also entrain most part of the snowcover laying on the ground. To investigate the influence of the entrained mass on the dynamics of large avalanches, model simulations with and without entrainment were performed.

During the Winter 1999, a large part of the northern flank of the Alps was struck by massive avalanches. Three very large avalanches events were artificially triggered at the VdlS test site. The largest avalanche was released on the 25 February. It was characterised by an average fracture height of about $1.95 \mathrm{~m}$ that extended over a length of about $1 \mathrm{~km}$. By the use of photogrammetric measurements, the total release mass was estimated to be 80000 tons. The deposition volume was determined too. The avalanche travelled more than $4000 \mathrm{~m}$. The image processing of a video recording gave the frontal speed of the event in the first $2000 \mathrm{~m}$ of track. FMCW radar, located in three positions along the avalanche path, allowed local entrainment analysis and distribution of flow height to be determined. The average height and density of the snowcover entrained by the avalanche have been approximated to be about $1.5 \mathrm{~m}$ and $200 \mathrm{~kg} / \mathrm{m}^{3}$, respectively.

In order to simulate a very large avalanche event with a one-dimensional model the spatial variability of the terrain profile, the frontal speeds, the flow heights and the deposit distributions should be carefully analysed in order to find the correct one-dimensional approximation.

As with the Pizzac avalanches, avalanche A3 is also back calculated using a Voellmy-Fluid model and a NIS model. Simulations with and without entrainment were performed. Figures 13,14 and 15 show the results of these simulations.

Simulations without entrainment (NIS model parameters: $m=0.003, b=0.34, s=0.4, v_{1}=0.001$ and $v_{2}=0.0001$ and VS parameters: $\mu=0.16, \xi=2700$ and $\lambda=2.5$ ) clearly show discrepancies: flow heights and depositions are strongly underestimated (see Figs. 14 and 15). It is important to point out that the model does not account for density variations. This means that the density of the avalanche along the avalanche path is the same density of the avalanche in the deposition zone. A normal density value used in the simulations is $300 \mathrm{~kg} / \mathrm{m}^{3}$. This density could be a reasonable value during the flow phase. However, observations of densities in large avalanche deposits shows a much higher value: 400$600 \mathrm{~kg} / \mathrm{m}^{3}$. Since the model does not consider snow compression, the calculated deposition height should be reduced by a $75 \%$ to $50 \%$.

Better simulations are obtained by introducing flow entrainment. Figure 14 shows flow height simulations using 


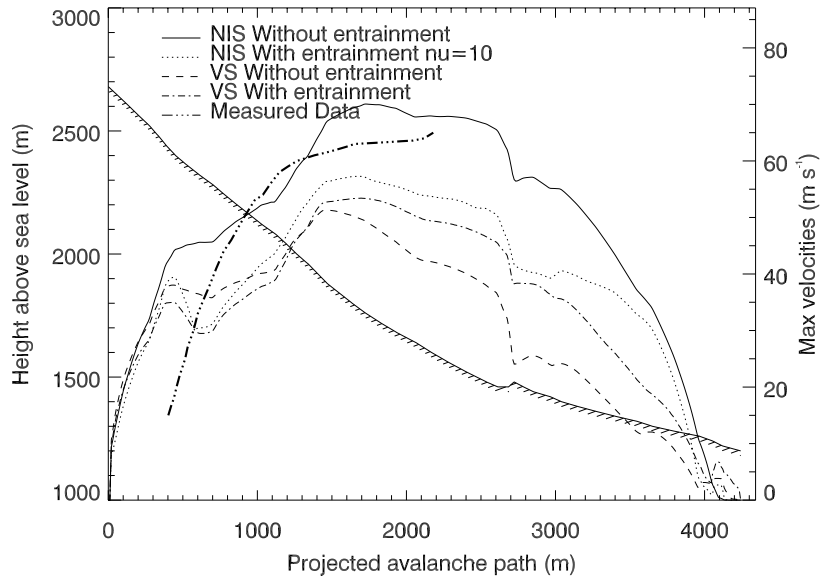

Fig. 13. Speed simulations of a large avalanche (Vallée de la Sionne event of 25 February 1999 using the Voellmy-fluid model (VS) and the NIS Norwegian model. Simulations without and with entrainment are compared to experimental data. Note that speeds calculated with the NIS model are in good agreement with experimental data.

the NIS model $\left(m=0.055, b=0.34, s=0.4, v_{1}=0.0001 v_{2}\right.$ $=0.1$ and $m=0.055, b=0.34, s=0.4, v_{1}=10$ and $\left.\nu_{2}=0.1\right)$ and the Voellmy-Salm (VS) model $(m=0.23, \xi=2500$ and $\lambda=2.5$ ).

The simulated avalanche entrains snow by eroding a userspecified snow cover. The snow cover is composed of one layer characterised by a height and density equal to the height and density of the layer entrained by the real avalanche. The average density value used for these calculations is $200 \mathrm{~kg} / \mathrm{m}^{3}$; the average entrainment height is $150 \mathrm{~cm}$. All the user-specified snow cover was entrained.

Simulations have been compared with the height-intensity output of a FMCW radar located along the avalanche path. The radar plot interpretation was difficult because the boundary layer between dense and powder part of the avalanche was not clear defined (we are simulating only the dense part of the avalanche). The maximum dense flow height was estimated in about $5 \mathrm{~m}$. The avalanche took more that 90 seconds to pass over the radar.

The better simulation is given by the NIS model with entrainment and a large longitudinal viscosity $v_{1}=10$ (see Fig. 14). Note that without the introduction of a high value of normal stress viscosity, height of flows are too high. Also with the introduction of a large longitudinal viscosity the avalanche length is too short; more mass should be moved back into the tail. This means that internal shear deformations together with the longitudinal straining defined in the model are not strong enough to account for the back-dislocation of the entrained snow in large avalanches. In large avalanches the process of mass transfer inside the avalanche body appears to be stronger than in small avalanches.

It should be considered that the model uses constant friction values along all the avalanche length. It has been demon-

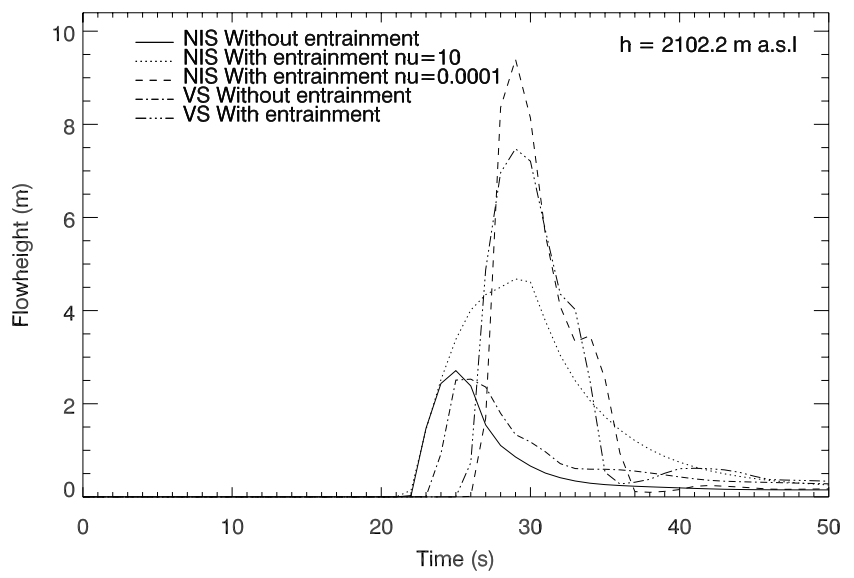

Fig. 14. Flow height simulations of a large avalanche (Vallée de la Sionne event of 25 February 1999) using the NIS model and the Voellmy-Salm (VS) model. Simulations are compared with the output of a FMCW radar located at the same position along the avalanche path. The radar plot interpretation suggests that the better simulation is given by NIS model with entrainment and $v_{1}=$ 10. Note that without the introduction of a high value of normal stress viscosity, height of flows are too high and avalanche length too short.

strated that the distribution of the mass within the avalanche is dependent on the terrain/snow cover friction. Higher friction increases the snow transfer from the body to tail of the avalanche (see Sect. 3.3(3)). In reality, the importance of the friction differs from avalanche tail, where roughness and avalanche height are comparable, and avalanche head, where avalanche dimensions are an order of magnitude larger in respect to the roughness. The application of a variable friction parameter could accelerate avalanche front and decelerate avalanche tail, simulating the natural extension of the avalanche.

Observations of deposition heights show that only with entrainment, real and calculated volumes in the deposition zone are similar (see Fig. 15). The simplified one-dimensional profile doesn't allow a precise deposit distribution to be obtained.

\section{Conclusions}

A quasi one-dimensional dense snow avalanche model, based on the Voellmy fluid flow law and a Norwegian NIS model, were modified by introducing entrainment and deposition. Simulations with and without snowcover entrainment have been performed.

Results show that models that do not consider entrainment can only be used for the determination of runout distances and, under certain limits, for frontal speeds. Calculated flow heights and deposition distributions are unrealistic. A comparison between experimental data and model simulations without entrainment shows that there are many discrepancies. The Voellmy-fluid flow model strongly underestimated 


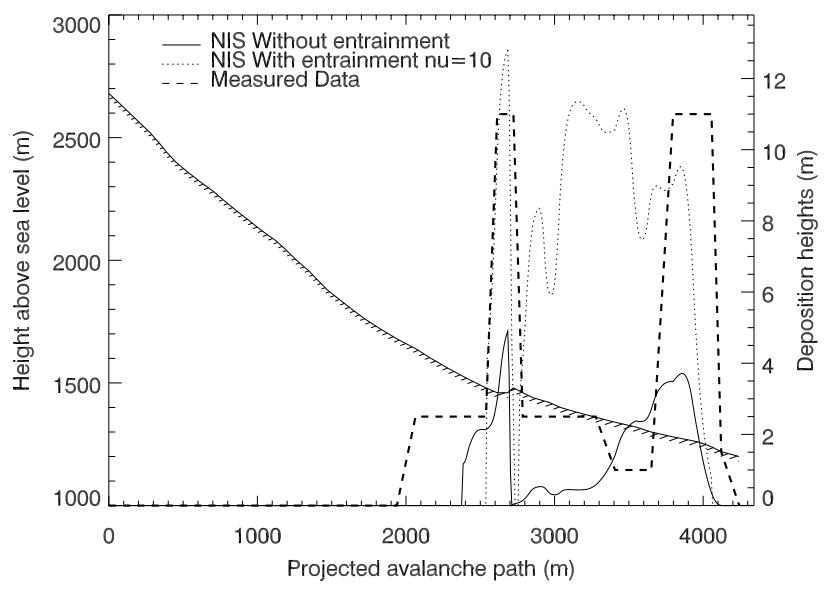

Fig. 15. Deposit simulations of a large avalanche (Vallée de la Sionne event of 25 February 1999) using the NIS Norwegian model. Simulations with and without entrainment are compared with experimental data. Since the model do not account for density variation, calculated heights should be reduced by a $75 \%$ to $50 \%$. (Calculation density $300 \mathrm{~kg} / \mathrm{m}^{3}$, deposit density $400-600 \mathrm{~kg} / \mathrm{m}^{3}$ ). Note that also for large avalanches entrainment is necessary in order to obtain the correct deposited mass.

frontal speeds and heights of flow. Deposition heights were also unrealistic. The NIS model matches the observed frontal speeds better, but, without entrainment, height of flow and deposition heights are an order of magnitude too small.

The introduction of the entrainment helps explain these discrepancies and allows more accurate simulation of the events. The influence of the entrained mass is important for both large and small avalanches.

The calculations show that the NIS model simulates both large and small avalanche events better. This suggests that models including internal shear deformation, i.e. not constant internal speed profile, are necessary in order to improve the accuracy of the model calculations.

Variable friction coefficients and density, along the avalanche length, could improve the determination of the frontal speed for small avalanches and flow heights for large avalanches.

It has been observed that it is not only important to establish the quantity of mass that an avalanche can potentially erode but also it is more important to determine how this mass is distributed into the avalanche body. The best modelling results were obtained when the entrained mass is accelerated up to the flow speed with a time delay (in agreement with Sect. $3.3(8)$ ). We have also shown that the distribution of the mass inside the avalanche, i.e. the time delay between the instant the mass is eroded and reaches the avalanche speed, can influence the dynamics of the avalanche more than the quantity of the entrained snow mass.

In future, the delay can be obtained analysing more events in order to find a constitutive law that describe the process.

Acknowledgement. We thank W. Ammann, A. Luchetta and F. Sommavilla for support this work, M. Christen, F. Leuenberger and all the avalanche dynamic team for the fundamental help. Funding is provided by the Swiss National Foundation.

\section{References}

Ammann, W. J.: A new Swiss test-site for avalanche experiments in the Vallée de la Sionne/Valais, Cold Regions Science and Technology, 30, 3-11, 1999.

Bartelt, P., Salm, B., and Gruber, U.: Calculating densesnow avalanche runout using a Voellmy-fluid model with active/passive longitudinal straining, J. Glaciol., 45, 242-254, 1999.

Bartelt, P. and Stöckli, V.: The influence of tree and branch fracture, overturning and Debris entrainment on snow avalanche flow, Ann. Glaciol., 32, 209-216, 2001.

Harbitz, C. B., Issler, D., and Keylock, C. J.: Conclusions from a recent survey of avalanche computational models, in: Hestnes, E. (Ed.): Proceedings of the Anniversary Conference 25 Years of Snow Avalanche Research, Voss, 12-16 May 1998, Oslo, Norwegian Geotechnical Institute (NGI Pubblication 203), 128-139, 1998.

Issler, D. (Ed.): European avalanche test sites. Overview and analysis in view of co-ordinated experiments, Eidg. Inst. Schnee-und Lawinenforsch. Mitt., 59 (SAME deliverable), 1999.

Gubler, H.: Measurements and modelling of snow avalanches speeds, IAHS, 162, 405-420, 1986.

Gubler, H. and Hiller, M.: The use of microwave FMCW radar in snow and avalanche research, Cold Regions Science and Technology, 9, 109-119, 1984.

Norem, H., Irgens, F., and Schieldrop, B.: Simulation of snowavalanche flow in run-out zones, Ann. Glaciol., 13, 218-225, 1989.

Perla, R., Cheng, T. T., and McClung, D. M.: A two-parameter model of snow-avalanche motion, J. Glaciol., 26 (94), 197-207, 1980.

Salm, B.: Contribution to avalanche dynamics, International Association of Scientific Hydrology Pubblication 69 (Symposium at Davos 1965 - Scientific Aspects of Snow and Ice Avalanches), 199-214, 1966.

Salm, B., Burkard, A., and Gubler, H.: Berechnung von Fliesslawinen: eine Anleitung für Praktiker mit Beispielen, Eidg. Inst. Schnee und Lawinenvorsch. Mitt., 47, 1990.

Sartoris, G. and Bartelt, P.: Upwinded finite difference schemes for dense snow avalanche modeling, International Journal for $\mathrm{Nu}-$ merical Methods in Fluids, 32, 799-821, 2000.

Savage, S. B. and Hutter, K.: The motion of a finite mass of granular material down a rough incline, J. Fluid Mech., 199, 177-215, 1989.

Schaer, M. and Issler, D.: Particle densities, velocities and size distributions in large avalanches from impact-sensor measurements, Ann. Glaciol., 32, 321-327, 2001.

Sommavilla, F. and Sovilla, B.: The avalanche monitoring system of Mount Pizzac, in: Hestnes, E. (Ed.): 25 Years of Snow Avalanche Research, Voss, 12-16 May 1998. Proceedings. Oslo, Norwegian Geotechnical Institute (NGI Pubblication 203), 268273, 1998.

Sovilla, B., Sommavilla, F., and Tomaselli, A.: Measurements of mass balance in dense snow avalanche events, Ann. Glaciol., 32, 230-236, 2001.

Vallet, J., Gruber, U., and Dufour, F.: Photogrammetric avalanche volume measurements at Vallée de la Sionne, Ann. Glaciol., 32, 141-146, 2001. 